\title{
Effect of Short-Term Fasting on Bone Elongation Rates: An Analysis of Catch-up Growth in Young Male Rats
}

\author{
CORNELIA E. FARNUM, ANDREA O. LEE, KATHLEEN O'HARA, AND NORMAN J. WILSMAN \\ Department of Biomedical Sciences, College of Veterinary Medicine, Cornell University, Ithaca, New \\ York, U.S.A. [C.E.F., A.O.L., K.O.]; Department of Comparative Biosciences, School of Veterinary \\ Medicine, University of Wisconsin-Madison, Madison, Wisconsin U.S.A. [N.J.W.]
}

\begin{abstract}
ABSTR
Bone elongation in the postnatal animal is a result of cellular
activity during endochondral ossification. Growth plate chondro-
cytes undergo a differentiation cascade involving stem cell clonal
expansion and cellular enlargement during hypertrophy. Nutri-
tional status has a significant effect on rates of bone growth, and
a period of accelerated growth will occur if nutritional stunting of
growth in early childhood can be corrected. This study focuses
on changes in rates of increase in bone length in a model of
catch-up growth in 4 -wk-old male rats. Animals fasted for $3 \mathrm{~d}$
reached a weight $\sim 60 \%$ of the control littermates. By $28 \mathrm{~d}$
postfasting, fasted animals had regained weight to $95 \%$ of control
levels. A 3 -d fast caused an immediate and profound decrease in
rate of growth in the proximal tibial growth plate to only $30 \%$ of
that of control animals, while stopping growth in the distal tibial
growth plate. During the rapid initial rate acceleration of bone
\end{abstract}
elongation, growth rate in both growth plates reached that of the control littermates by $7 \mathrm{~d}$ postfasting. The proximal tibial growth plate then maintained rates that were $10-15 \%$ higher than control over the rest of the experimental period. By $10 \mathrm{~d}$ postfasting, the previously fasted animals were on the same weight/rate trajectory as the control littermates. Changes in elongation rates were reflected by dramatic changes in growth plate morphology in all cellular zones. This is the first study to directly correlate weight recovery during catch-up with growth rate responses at the level of the growth plate. (Pediatr Res 53: 33-41, 2003)

OTC, oxytetracycline

PTU, propylthiouracil

RHT, ruthenium hexamine trichloride
In both the prenatal and the postnatal animal, there is a complex interplay between overall nutritional status and linear bone growth. There is experimental evidence that GH/IGF-1 concentration is responsive to changing nutritional status (15), and growth responses to food deprivation also have been shown to involve the hormone leptin, which is secreted by adipocytes (6-8). However, how undernutrition is translated into changing cellular activity during bone elongation has not been studied. This is significant for understanding cellular mechanisms behind the complex phenomenon of catch-up growth, which can occur to different extents, and at different rates, depending upon whether nutritional deprivation occurs in utero, during the early neonatal period of nursing, or later in postnatal growth $(4-6,9-12)$. Catch-up growth, as first described by Prader in 1963 (13), is characterized by a period of

Received March 19, 2002; accepted August 16, 2002.

Correspondence: Cornelia E. Farnum, D.V.M., Ph.D., Department of Biomedical Sciences, T5 010-D Veterinary Research Tower, College of Veterinary Medicine, Cornell University, Ithaca, NY 14853, U.S.A.; e-mail: cef2@cornell.edu

Supported by National Institutes of Health grant AR 35155 to N.J.W. and C.E.F.

DOI: 10.1203/01.PDR.0000041518.63329.FF growth accelerated above normal during recovery from a period of previous deprivation, which allows the child to accelerate toward, and even resume, his/her preillness growth curve (14). A recent definition is "height velocity above the statistical limits of normality for age and/or maturity during a defined period of time, following a transient period of growth inhibition (9)."

Multiple rodent models of catch-up growth have been investigated, differing in the timing of, and the nature of, the stimulus for growth retardation. The most common pharmacologically induced models to rapidly slow growth use either the anti-thyroid agent PTU (15-17) or dexamethasone (5, 14, 17-19). Models in which manipulation of nutrition is the primary variable include 1) food restriction to the mother during gestation and/or lactation $(20,21)$; 2) bilateral uterine artery ligation during late gestation $(22,23)$; 3 ) manipulation of litter size during weaning, contrasting litters of four pups to litters of 16 or more pups $(11,18,24)$; and 4) short periods of fasting to the postweaning animal (3, 5, 17, 25-29). These experiments have demonstrated that the timing and the duration of the stimulus are critical, with differing outcomes in 
prenatal/postnatal and preweaning/postweaning experiments (27).

The purpose of this study was to analyze changes in long bone growth rates at the level of the growth plate in shortterm-fasted rats post refeeding over a 28-d interval. The model was 4-wk-old male rats in pairs with one having a 3-d fast and the littermate used as a control. This is a model intended to understand growth plate cellular response to, and catch-up of bone elongation after, a period of short-term malnutrition and/or total food deprivation during the rapid growth phase of childhood/adolescence. Growth rates in the proximal and distal tibial growth plates were measured using the fluorescent calcium chelator, oxytetracycline, which allows an estimate of the rate of growth in the 24-h period before euthanasia (30). Body weights and food consumption in the catch-up period also were monitored. The hypothesis was that 24-h rates of tibial growth initially would be significantly depressed in the fasted animals compared with control littermates, but that rates of growth would increase until they exceeded that of control littermates during the time course of catch-up growth. It also was hypothesized that the timing of growth plate closure would not be affected in this model. The data also allow a comparison of the trajectory of catch-up in weight to the trajectory of catch-up in rates of bone elongation, and how both of these are sustained through time.

\section{METHODS}

Sprague-Dawley rats (CD-1 IGS) were purchased from Charles River Laboratories and a small breeding colony was established at our facility for the rats used in this study. To ensure that intrauterine and preweaning litter size were not variables, only rats born into litters sizes of 10-14 animals were used. The rats were weaned at $3 \mathrm{wk}$ and fed $a d$ lib until $4 \mathrm{wk}$ of age (peripubertal). Mothers were fed a pregnancy chow [Global 22/5 Rodent Diet (W) 8640, Harlon Teklad, Madison, WI, U.S.A.] during both pregnancy and nursing. All animals were kept under circadian lighting conditions, with $12 \mathrm{~h} \mathrm{light} / 12 \mathrm{~h}$ dark. Male littermates were assigned to groups as pairs, one to continue to be fed a commercial rat diet (Teklad Global 16\% Protein Rodent Diet, 2016) ad libitum (control), and the other to be fasted for $3 \mathrm{~d}$ with water provided (fasted). All animals had access to a large tube for shelter in each individual cage. During the fasting period, the animals were observed daily both by ourselves and by a staff veterinarian; the fasted rats remained bright, alert, and active. There was no evidence of spontaneous disorders in any animal throughout the study. Male rats were used because it is well documented that there is a sexual dimorphism in the response to $\mathrm{GH}$, male rats gain weight faster than female rats in the postweaning period, and male rats reach a final large body size (26, 30-32).

Time zero was defined as the end of the fast, and collections of proximal and distal tibial growth plates were made at 0,1 , $2,3,7,14,21$, and $28 \mathrm{~d}$. Each time group consisted of six animals from different litters. This experimental design involved 96 animals total (48 controls and 48 fasted) representing 22 litters. Animals were injected with the calcium chelator OTC $(5 \mathrm{mg} / \mathrm{kg}) 24 \mathrm{~h}$ before euthanasia. OTC is laid down initially in areas of high calcium turnover, which includes the calcified septa of the growth plate in the distal hypertrophic cell zone and the newly formed metaphyseal bone (33). Incorporated OTC after $24 \mathrm{~h}$ can be viewed as a fluorescent band in the metaphyseal bone. All procedures were approved by the Institutional Animal Care Committee.

The two growth plates of the tibia were chosen for analysis. In the 4-wk-old rat, the proximal tibial growth plate and is one of the fastest growing in the animal, and it is growing at approximately three times the rate of the distal tibial growth plate (34). In addition, the proximal growth plate will continue in active growth for several weeks longer than the distal growth plate (35). Therefore, these two growth plates are representative of differential growth rates occurring in the animal both at one point in time and through time.

Growth plate collections were made $1 \mathrm{~h}$ after lights on. After euthanasia with a lethal dose of pentobarbital $(300 \mathrm{mg} / \mathrm{kg}$, i.p), the two growth plates were collected, rapidly trimmed, and fixed for $3 \mathrm{~h}$ in $2 \%$ paraformaldehyde $/ 2 \%$ glutaraldehyde in $0.1 \mathrm{M}$ cacodylate buffer, $\mathrm{pH} 7.3$, with $0.7 \%$ RHT, and embedded in Epon-Araldite using standard techniques (34). RHT is a small molecular weight cationic dye that diffuses rapidly into the cartilaginous matrix, stabilizing both the negatively charged proteoglycans of the matrix as well as the interface between the chondrocytic plasma membrane and the pericellular matrix. RHT has been demonstrated to be required for satisfactory preservation of growth plate chondrocytes similar to their in vivo morphology, and therefore RHT-fixed growth plates are considered to give optimal fixation (36).

OTC fluorescence was visualized under epifluorescent microscopy on 1-micron-thick sections with an excitation filter of $365-380 \mathrm{~nm}$ and a barrier filter of $530-540 \mathrm{~nm}$. To estimate the elongation over $24 \mathrm{~h}$, the distance between the leading edges of the fluorescent band in the metaphyseal bone and the chondro-osseous junction was measured on video-captured images (3CCD color Sony Progressive video camera, Sony, Teaneck, NJ, U.S.A.). The chondro-osseous junction was identified and a line was drawn traversing it. Next, the leading edge of the fluorescent OTC band was identified, and a second line drawn that represented its location in the metaphyseal bone. Both of these lines were drawn with a relatively thick drawing tool such that each line could represent the general form of the transition it was identifying, minimizing minor irregularities. Elongation was determined by averaging 10 measurements equally divided from sections from two different blocks from the same animal. Measurements where made by individuals blinded as to the specific identity of the slide they were measuring, to eliminate the potential for systematic bias. We have found this method of measurement to give consistent results among different individuals, and we as well as others have used it in previous experimental situations $(34,35,37-$ 39). Color staining of one-micron-thick sections was with basic fuschin/methylene blue/azure 2 (40).

Animals were weighed five to seven times a week from d 0 throughout the experimental time period. Food consumption was monitored at similar time intervals. For all subsequent calculations, differences between groups were analyzed by ANOVA, at $p<0.05$, with a posteriori pair-wise comparisons. 


\section{RESULTS}

A 3-d fast led to a dramatic weight difference between the control and fasted group, as seen in Figures 1 and 2. Starting from initial weights that were essentially identical, control rats increased in weight by $16.7 \%$ (102.4 $\mathrm{g}$ at $\mathrm{d}-3$ versus $119.5 \mathrm{~g}$ at $\mathrm{d} 0$ ), whereas fasted rats lost weight by $29.8 \%$ during the fasting period ( $104.0 \mathrm{~g}$ at $\mathrm{d}-3$ versus $73.0 \mathrm{~g}$ at $\mathrm{d} 0$ ). Thus, by the end of the 3-d fasting period, weights of the fasted animals averaged only $60.0 \%$ of their matched littermates $(p<0.01)$. Weight gain as a function of time postfasting is shown in Figure $3 \mathrm{~A}$. Fasted animals weighed less than control animals throughout the experimental period, but the magnitude of the difference continued to lessen over time. Starting at weights $60 \%$ of their control littermates immediately postfast, the fasted animals reached $95 \%$ of the control weight after $28 \mathrm{~d}$. The differences in weight were statistically significant at $p \leq$ 0.02 for each of the time periods, except at $28 \mathrm{~d}(p=0.06)$. In Figure $3 B$, cumulative percent weight gain is plotted against

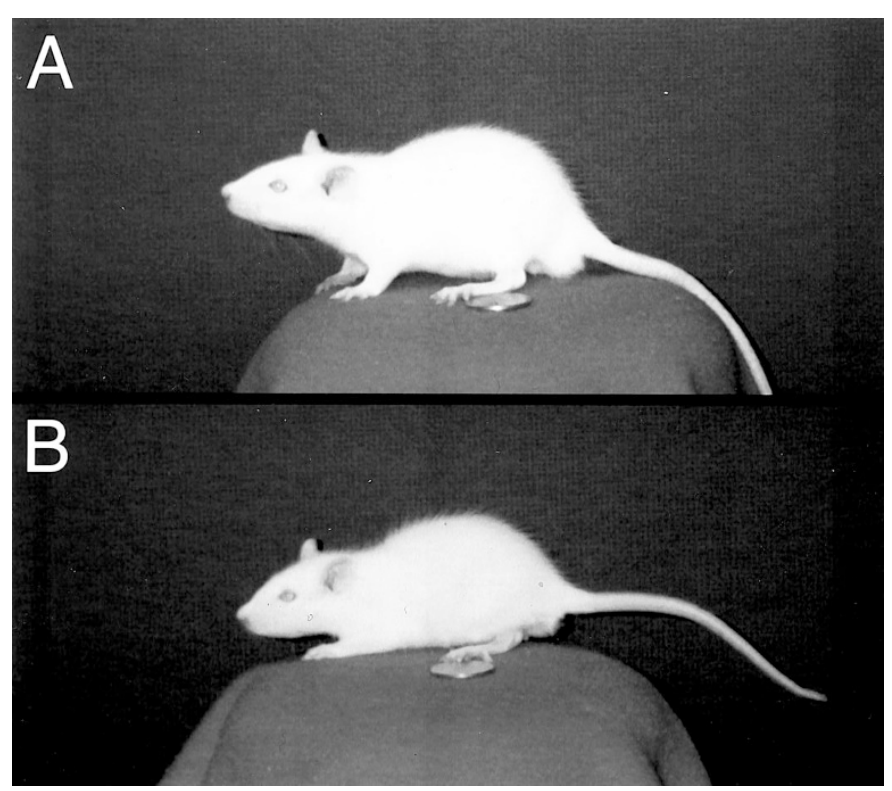

Figure 1. Size effects of a 3-d fast. The rats in $A$ and $B$ are male littermates. $A$ shows a control animal and $B$ is his littermate, who was fasted for $3 \mathrm{~d}$ at 4 wk of age. Photos were taken immediately after the fasting period.

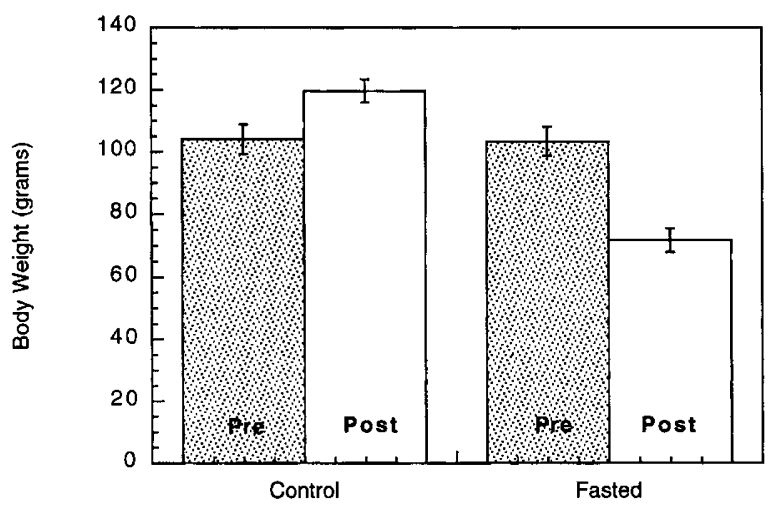

Figure 2. Weight effects of a 3-d fast. At $4 \mathrm{wk}$ of age, all rats weighted slightly over $100 \mathrm{~g}$. After a 3 -d fast, the fasted animals averaged only $\sim 60 \%$ of the weight of the control animals. days from the end of the fast. Over the 4-wk experimental period, control animals increased in weight $\sim 275 \%$; in the same period of time, fasted animals increased in weight by $\sim 450 \%$. For the first $3 \mathrm{~d}$ of the experimental period, daily weight gain for the fasted animals averaged $12.4 \pm 1.3 \mathrm{~g}$ compared with $8.6 \pm 0.8$ for the controls $(p<0.01)$. By comparison, for the last $3 \mathrm{~d}$ of the experimental period, the weight gain for the fasted animals $(6.3 \pm 1.6 \mathrm{~g})$ was not statistically different from the controls $(5.2 \pm 2.4 \mathrm{~g})(p=$ $0.30)$.

Figure 4 plots food consumed per day in relationship to body weight for the two groups throughout the experimental period. For the first $2 \mathrm{wk}$, the fasted animals consumed more food in relationship to body weight than did the controls; at the time points of 3 and 4 wk, the rates for the two groups were identical. Therefore, during the period in which the most rapid changes were occurring, the previously fasted animals were consuming significantly more food for their body size than were controls.

Figure 5 demonstrates the method by which growth rates were calculated from histologic sections showing OTC florescence. On each section, the chondro-osseous junction of the growth plate was identified (proximal arrowhead in the left margin of each micrograph). The distance from the chondroosseous junction to the leading edge of the fluorescent band (distal arrowhead) represented growth over the last $24 \mathrm{~h}$. It is readily seen in Figure 5, comparing the proximal tibial growth plate from a control animal at 0 time to the proximal tibial growth plate from a fasted animal at 0 time, that there was a significant retardation of growth rates as a result of fasting, a reduction to a rate that was $\sim 30 \%$ of control immediately after the fasting period.

Actual growth rates for both the proximal and the distal tibial growth plates at all time periods are plotted in Figure 6. In the control animals starting at $d 3$, there is a clear constant slowing of growth rate in the proximal growth plate over the experimental time period, from $\sim 350 \mu \mathrm{m} / \mathrm{d}$ and declining to $\sim 180 \mu \mathrm{m} / \mathrm{d}$. This represents a decline of approximately $50 \%$ over the 4-wk period. These rates agree well with previously recorded rates for this growth plate and demonstrate the high but steadily declining rate of growth that is characteristic of rodent adolescence $(30,34,35)$. The distal tibial growth rate mirrors this picture, albeit beginning at a rate less than one third of the proximal growth plate and effectively ending statistically significant growth $21 \mathrm{~d}$ into the experimental pe$\operatorname{riod}(7 \mathrm{wk}$ of age), with rates that are just at the limit of reliable measurement $(<15 \mu \mathrm{m} / \mathrm{d})$.

Rates of growth in the proximal tibial growth plate of the fasted animals began at approximately one third of that of controls immediately postfasting, and actually fell by the first postfasting (difference control versus fasted $p<0.01$ for all four time points) (Fig. 6). Then, however, there was a very steep rise to the growth rate curve so that by $\mathrm{d} 7$ the rate was identical to that of the control animals $(p=0.43)$. For the remainder of the experimental period, the growth rate of the proximal tibia remained above that of the control, sometimes by as much as $15 \%(p<0.03$ at $21 \mathrm{~d})$. Even after $28 \mathrm{~d}$, the proximal tibial growth plate of the fasted animals was still 

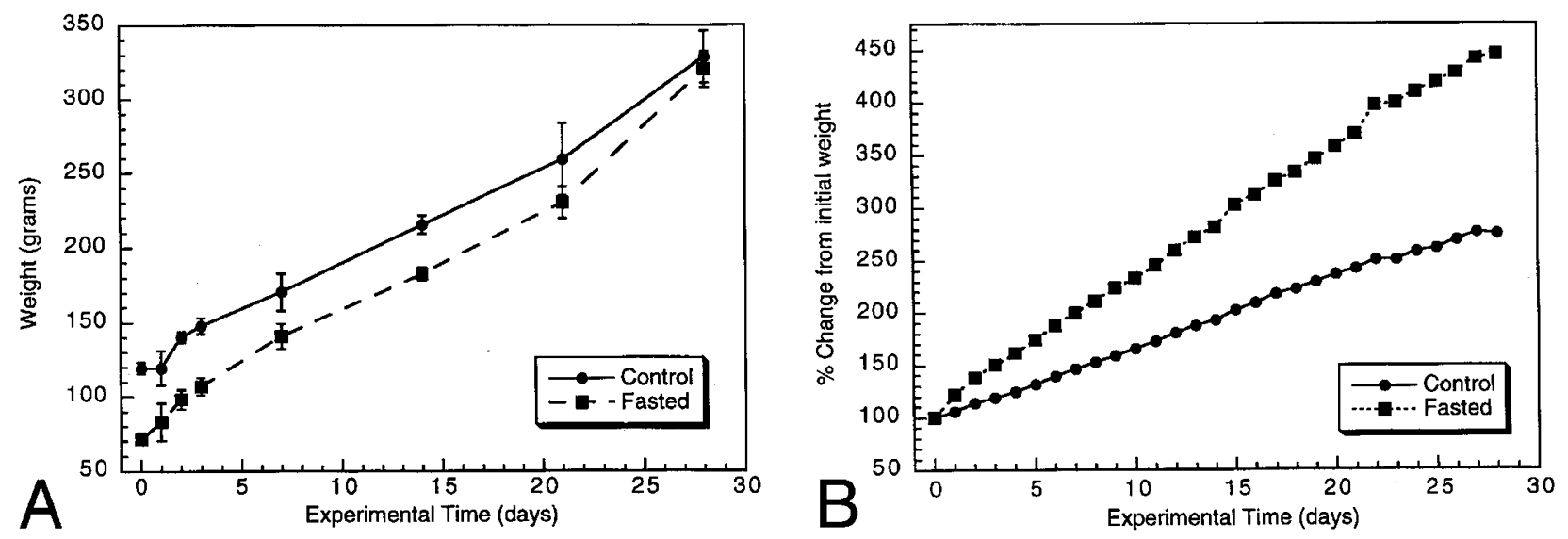

Figure 3. Weight gain of fasted and control rats. $A$ plots the weight gain in both control and fasted animals over a 4 -wk period postfasting. Immediately after the fast, fasted animals weighed approximately $60 \%$ as much as their control littermates compared with $95 \%$ as much at the end of the 4 -wk experimental period. $B$ demonstrates that control animals increased in weight by $\sim 250 \%$ over the $4 \mathrm{wk}$, whereas fasted animals increased their weight by over $425 \%$ in the same time period.

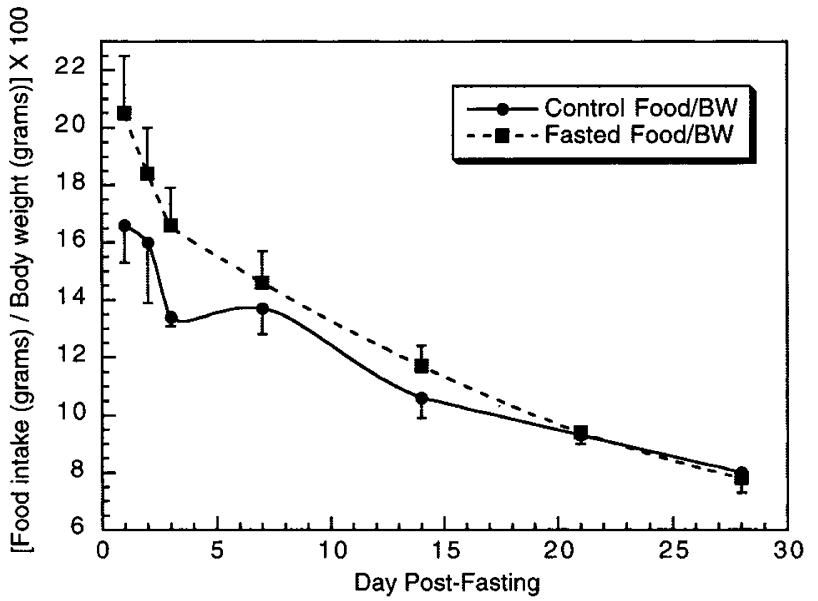

Figure 4. Weight gained to food consumed: comparisons of fasted and control animals. For the first 2 wk, fasted animals consumed more food per day in relationship to their body weight than their control littermates. However, by wk 3, food consumption in relationship to body weight of the fasted animals was identical to that of control animals.

growing $>10 \%$ over that of the controls, which is as much as $25 \mu \mathrm{m}$ of additional daily growth $(p<0.06)$. This is shown on the micrographs of Figure $7 A$, which demonstrate typical OTC data for the proximal tibial growth plate of control and fasted animals at $28 \mathrm{~d}$ postfasting. However, it should be noted that never during the catch-up period did rates in the proximal tibia of fasted animals reach the level of controls at their fastest.

Data for the distal growth plates as shown in Figure 6 mirror the findings for the proximal growth plate with two important exceptions. First, at d 0, essentially all growth had stopped in the distal tibial growth plate of the fasted animals (difference fasted from control, $p<0.002$ ). Secondly, although growth rapidly resumed and actually was above control by $\mathrm{d} 7(p=$ 0.10 ), growth in this growth plate approximated control values by $\mathrm{d} 21$ and, like that in the control animal, essentially was 0 by the end of the 28-d experimental period (difference fasted from control, $p=0.22$ ). Although it might be premature to generalize based on the data from this one growth plate, these data would suggest that catch-up occurred because of higher

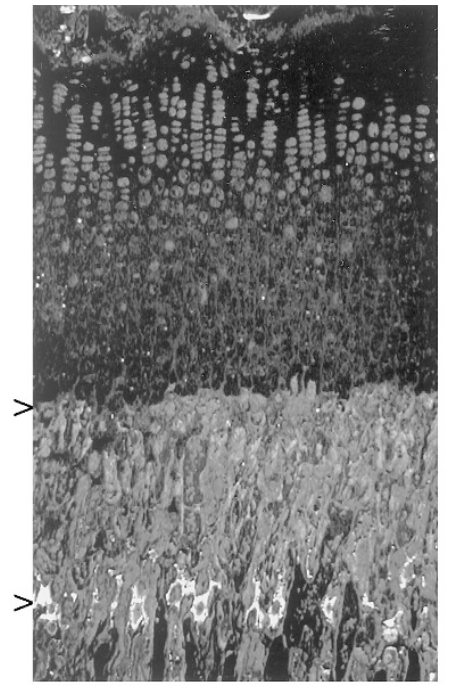

Control

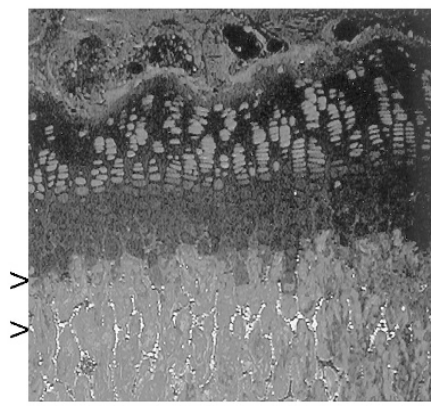

Fasted
Figure 5. Growth rates in the proximal tibia measured by OTC fluorescence. The method for measuring growth rate by OTC fluorescence by identifying the distance between the chondro-osseous junction of the growth plate (proximal arrow in each micrograph) and the leading edge of the fluorescent band seen in the metaphyseal bone is demonstrated. This distance represents the amount of growth in $24 \mathrm{~h}$. A comparison of the proximal tibial growth plate from a control animal after the 3-d fasting period with that from a fasted animal shows that fasting had reduced growth in this growth plate to approximately one third of normal. Bar $=100 \mu \mathrm{m}$.

rates immediately after the fasting period but without altering the timing of cessation of growth. The micrographs in Figure $7 B$ show this comparison: a control distal tibial growth plate at the left in Figure $7 B$ compared with the same from the fasted animal (center). The latter shows all florescence at the chondro-osseous junction, indicating cessation of growth. The micrograph to the right in Figure $7 B$ demonstrates the resumption of growth in the distal tibial growth plate of a fasted animal by d 7, to a value actually higher than that of the control at the same time period (latter not shown).

Figure $8 A$ compares the response trajectories for weight and proximal tibial growth rate in the fasted and control groups by plotting the ratio of fasted/control for these two variables as a 


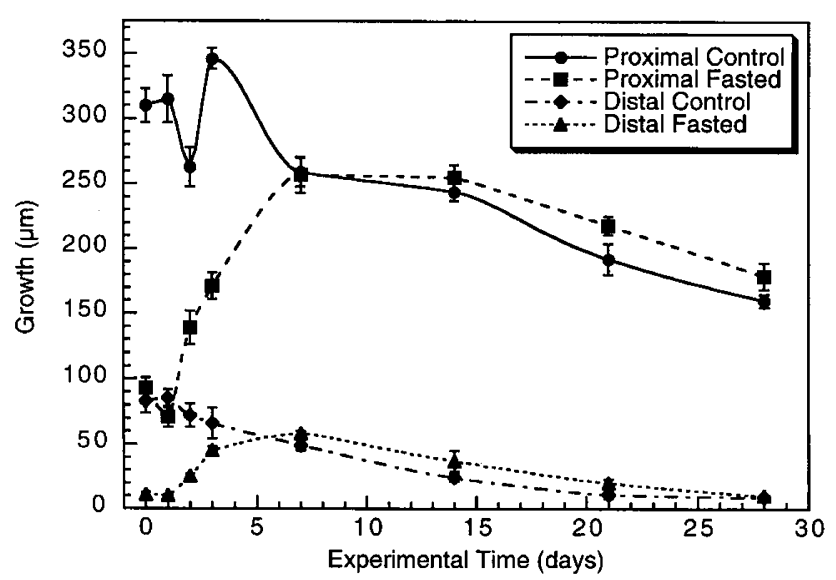

Figure 6. Comparisons of 24-h growth rates in growth plates from control and fasted animals. Growth rate of the proximal tibia in fasted animals, although beginning at a level $30 \%$ that of control animals, reached the level of controls by the end of the first week and remained $10-15 \%$ higher than control for the remainder of the experimental period. Results for the distal tibial growth plate mirrored this but were more dramatic. Fasting caused cessation of growth in the distal growth plate, growth rate caught up before $\mathrm{d} 7$, and by $\mathrm{d}$ 21 there was no statistical difference in rate between control and fasted animals. In both groups, the distal tibial growth plate essentially had stopped growing by wk 3 of the experimental period.
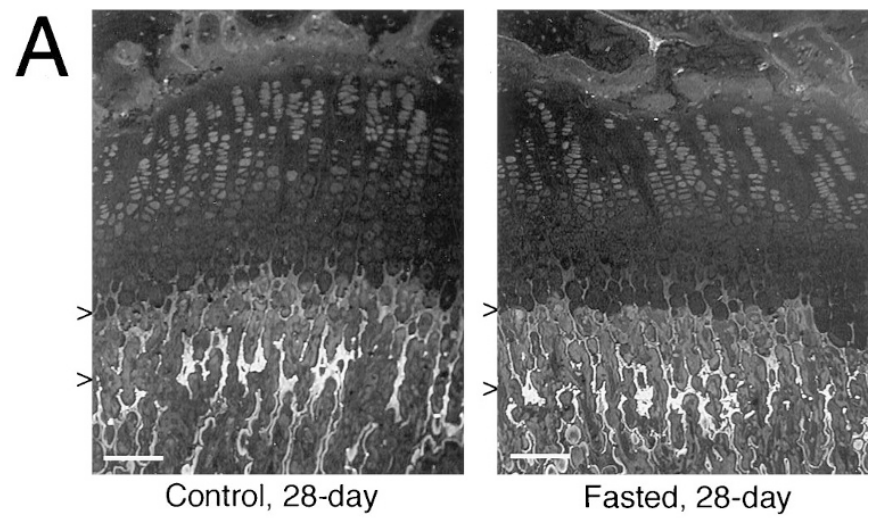

Fasted, 28-day
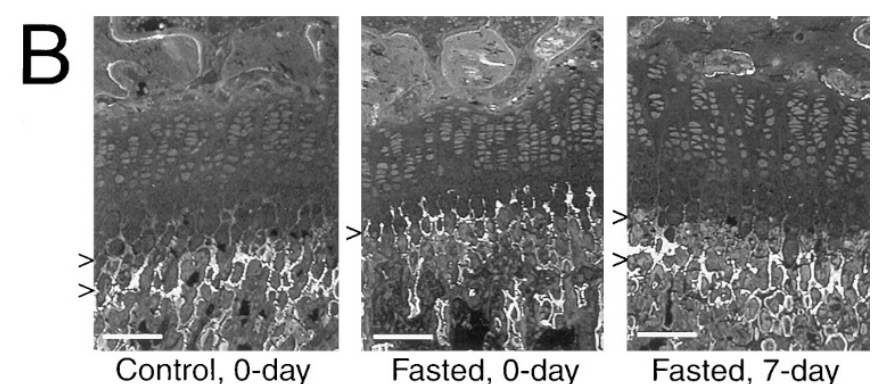

Figure 7. Growth rates in fasted and control animals over time by OTC fluorescence. $A$ demonstrates that, by the end of the experimental period, growth rate had slowed dramatically in both groups, but remained slightly higher in the previously fasted animals. $B$ makes important comparisons for the distal tibial growth plate. It is clear that immediately after the fast, growth in the distal tibial growth plate had ceased in the fasted animals, whereas there was significant growth in the controls. By d 7, growth had resumed in the distal tibial growth plate of fasted animals. Bar $=100 \mu \mathrm{m}$.

function of time. Although in the most literal sense the fasted animals never completely caught up in weight to the controls, in actuality they went from a weight that was approximately $60 \%$ of that of controls to a weight that was approximately
$95 \%$ of controls. Growth rate in the proximal tibia was reduced to approximately $30 \%$ of control after $3 \mathrm{~d}$ of fasting, but caught up to control rates in only $7 \mathrm{~d}$. It remained at a rate higher than controls for the rest of the experimental period. Figure $8 B$ plots the correlation between animal weight and rate of growth in the proximal tibial growth plate. The curve for the controls demonstrates that, as expected, as animals increase in weight, the rate of bone elongation in the growth plate decreases. For the fasted animals, in contrast, during the catch-up phase as weight increases, rate of bone elongation also increases. There is then an abrupt change when the trajectory of the fasted animals joins that of the controls, as weight increases and rate of elongation decreases in the last $3 \mathrm{wk}$ of the experiment. The growth trajectory for the two groups of animals is essentially identical during the last $3 \mathrm{wk}$ (superimposed curves), despite their differences in weight.

The histologic appearance of growth plates from pairs of animals at different time periods are illustrated in Figure 9, with control on the left in each figure and fasted on the right. Figure $9 A$ (left) demonstrates the general histologic organization of the proximal growth plate in control animals at the start of the experiment, showing the characteristic columnar arrangement of cells in a differentiation cascade of proliferation, hypertrophy, and cellular death with vascular invasion at the chondro-osseous junction. In animals fasted for $3 \mathrm{~d}$ (Fig. 9A, right) there was a very dramatic reduction in overall growth plate height, which included height changes in all cellular zones. Not only was there an apparent reduction in overall cellular number, but also in the final volume of hypertrophic chondrocytes. No disorganization of the characteristic spatial organization of growth plate chondrocytes was seen in growth plates from fasted animals. Figure $9 B$ demonstrates the similar comparison for the distal tibial growth plates. Distal growth plates in the two groups do not differ as dramatically, but in the fasted animals there is still a clear reduction in overall growth plate height, with an apparent decrease in cellular number and hypertrophic cell volume. Although not an absolute correlate, the overall difference in height between the proximal and distal tibial growth plates of the control animals reflects their very different growth rates, with the proximal growth plate growing at approximately three times the rate of the distal growth plate $(34,41)$. By d 7 , growth plates from the fasted and the control animals were growing at the same rate, and this was mirrored by their histologic appearances. The proximal growth plate of the fasted animals was essentially identical morphologically to that of the controls (Fig. 9C), and this was also true of the distal growth plates (Fig. 9D).

\section{DISCUSSION}

Whereas most studies follow catch-up growth in relationship to body weight or overall body length, many fewer studies have looked at the catch-up response specifically in relationship to the rate of linear growth of long bones of the limbs (14, $16,42)$. Postnatal bone growth occurs by endochondral ossification in growth plates at the ends of long bones. Within the growth plate, the spatial organization of chondrocytes reflects a temporal progression of chondrocytic differentiation (life 

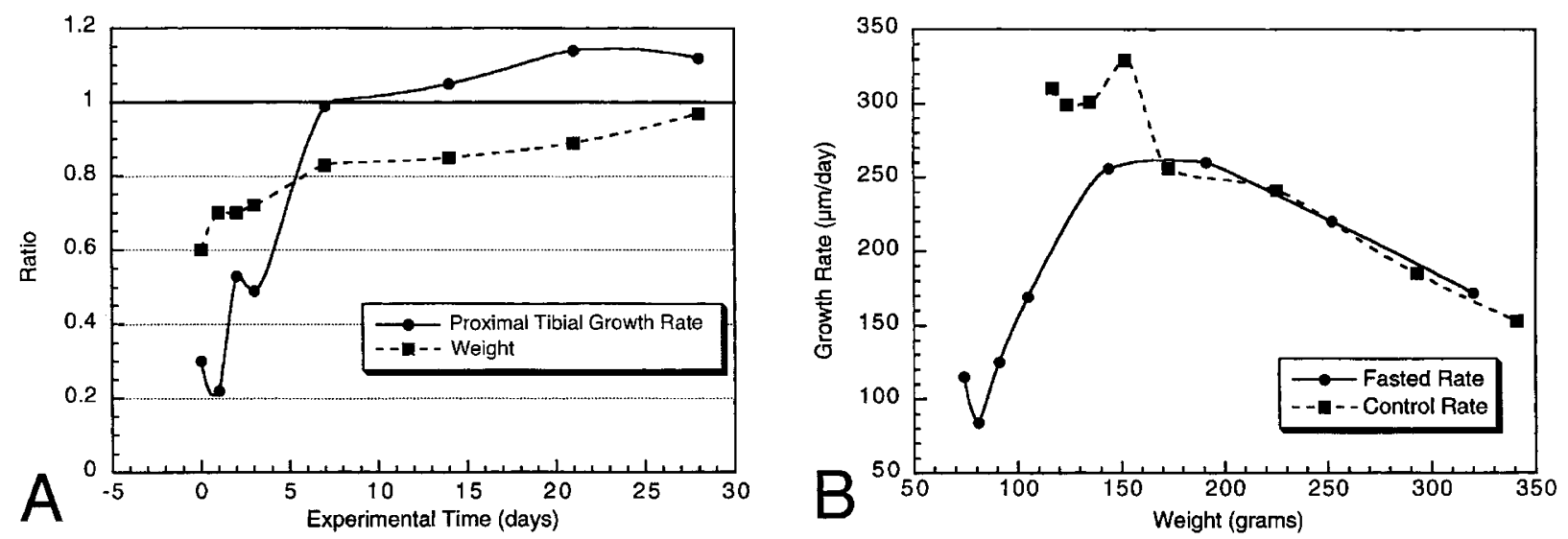

Figure 8. Growth rate in the proximal tibial growth plate and animal weight. $A$ plots ratios of fasted to control animals for weight gain and gain in growth rate in the proximal tibia during catch-up growth. Maintenance of a growth rate consistently higher than controls means that tibial elongation is still continuing. $B$ plots the correlation between growth rate in the proximal tibia and animal weight over the experimental period. As control animals gained weight, their rate of growth generally slowed. This was most apparent for the last $3 \mathrm{wk}$ of the experiment. However, fasted animals showed two trajectories of bone elongation rate. During the catch-up period (ascending in $B$ ), rate of growth increased as body weight increased. For the last 3 wk of the experiment, the trajectory of the fasted animals essentially mirrored that of controls.

history). The two primary rate dependent variables that interact to determine quantitative increases in long bone growth are mitotic activity in the proliferative zone and cellular volume increase and shape change during chondrocytic hypertrophy $(34,35,37,43)$. Conceptually these can be considered to be bounded by three important gates - the transition from the germinal chondrocyte's entrance into the cell cycle to start clonal expansion in the proliferative zone; the transition between the exit from the cell cycle to the initiation of cellular volume increase in the hypertrophic zone; and the transition from the terminal chondrocyte to vascular invasion (44). At each of these transitions there are major changes in gene expression. Rates of linear bone growth can be altered both by rate changes during proliferation and hypertrophy, as well as by rate changes at any of the three transition points $(44,45)$.

During the chondrocytic differentiation cascade that results in increased linear growth, there is a complex interplay between systemic, autocrine, and paracrine regulators of overall rate of growth. This is played out on the growth plate template that was patterned early in prenatal development and that has intrinsic capabilities and limitations, as well as a limited life span (closure occurs at the end of adolescence). Therefore, the growth plate is a particularly interesting organ to analyze in relationship to catch-up growth, not only because it is the organ that controls the rate and extent of linear growth, but also because it represents an organ where multiple levels of regulation, from that of embryological patterning to that of systemic and nutritional regulators at the level of the whole animal, are integrated.

This is the first study to measure precise growth rate responses to short-term fasting at the level of individual growth plates, rather than weight changes and/or changes in overall body or limb length. Growth rate in the proximal tibial growth plate of the fasted animals reached that of the control limb at 1 wk postfasting, and remained sustained at a higher rate of growth throughout the 4-wk experimental period. Therefore, the initiation of the catch-up response can be considered immediate and profound. Fasting led to immediate changes in cellular activity in the growth plate, such that longitudinal growth rate was reduced to approximately one third of control level in the proximal growth plate, and was essentially brought to zero in the distal growth plate. By examining two growth plates growing at very different rates, it is possible to generalize that the response is through an immediate change in growth rate at both ends of the limb, and therefore is a response that should be considered in light of what is known about the control of differential limb growth in the postnatal animal (34, $37,43,45)$.

Previous investigators, using several different fasting-based models of catch-up growth in which weight changes have been measured as the end point, have concluded that deprivations postweaning are much less serious than those preweaning, and that there is progressively less effect as the expected rates of growth in controls decreases $(27,28)$. Therefore, when examining the magnitude of the response in the present study, it is important that comparisons be made with other investigations in which the age, sex, species (and even strain) of the animals, and duration of the fast, are comparable. The percent weight loss that we recorded after a 3-dfast (29.8\%, starting with male rats weighing $\sim 100 \mathrm{~g}$ ) was comparable to that seen in several previous similarly designed studies: $25 \%$ loss, starting at weights of $\sim 120 \mathrm{~g}(26) ; 42.8 \%$ loss, starting at weights of $\sim 70$ $\mathrm{g}$ (17); and $28 \%$ loss, starting at weights of $\sim 125 \mathrm{~g}$ (16). Thus, this model is one of acute and severe nutritional deprivation, in which we have demonstrated that changes in bone growth rates are even more dramatic than changes in rates of weight gain/ loss. Its significance for disease in children is to further the understanding that growth plates respond to any severe period of nutritional stress. Recovery of bone elongation rates to control levels is rapid; however, recovery in terms of achieving the prestress predicted total length of the bone is a prolonged process, even if the period of insult was of very short duration.

All variables capable of altering rate of growth must be considered as potential mediators of the catch-up response. 
A

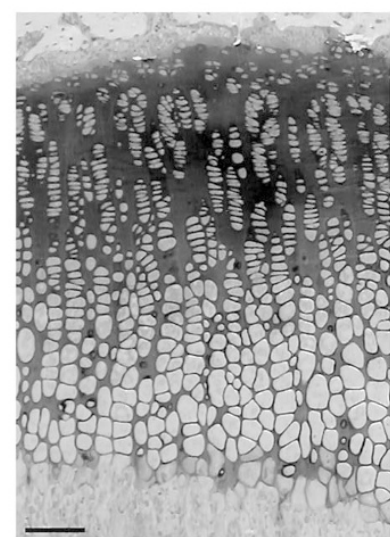

Control, PT
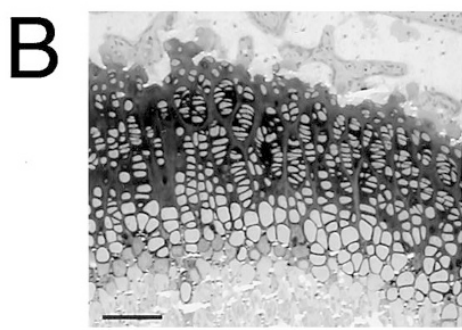

Control, DT
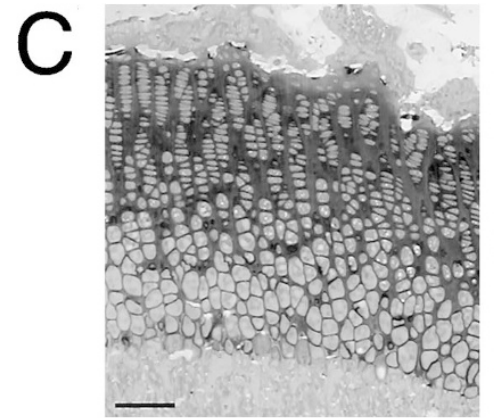

Control, PT
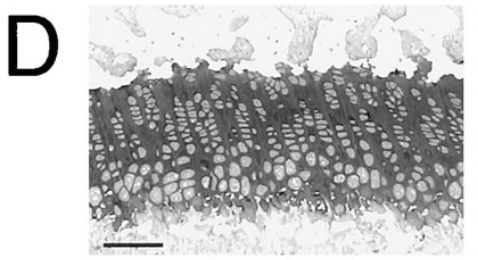

Control, DT

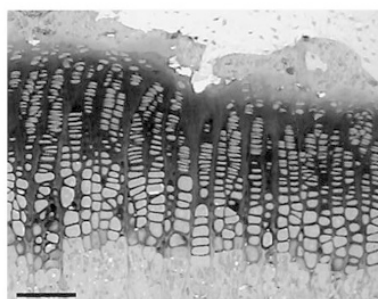

Fasted, PT

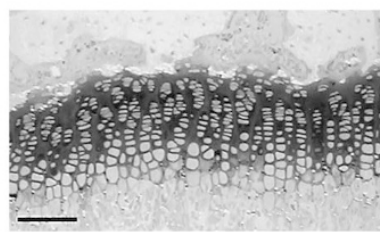

Fasted, DT

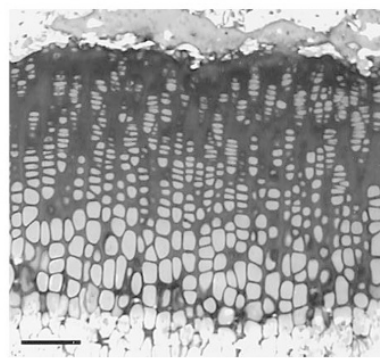

Fasted, PT

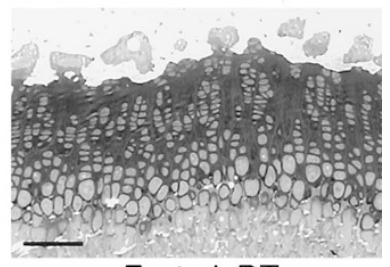

Fasted, DT

Figure 9. Histologic appearance of growth plates from control and fasted animals. $A$ shows the highly organized columnar arrangement of chondrocytes of the proximal tibia of a 4-wk-old control animals. In fasted animals, columnar organization was maintained. However there was a sharp reduction in overall growth plate height, with an apparent reduction in the number of chondrocytes in all zones. There was also an apparent reduction in the final volume of hypertrophic chondrocytes. $B$ makes a similar comparison for the distal tibial growth plates. $C$ and $D$ show the same comparisons for control and fasted littermates at $\mathrm{d} 7$, when catch-up in growth rate had been achieved. Bar $=100 \mu \mathrm{m}$.

These may vary depending upon the specific model. In a recent study using a dexamethasone model in rabbits, the primary alteration to the growth plate is reported to be to the proliferative cell population $(14,42)$. Therefore, the catch-up response in this model is consistent with hypothesizing that dexamethasone essentially causes a delay in, but not an alteration of, the

number of stem cell divisions. This has been interpreted as delaying senescence of the growth plate but not altering its total proliferative capacity, consistent with the concept of replicative senescence described in other cell types $(46,47)$. When dexamethasone was given to one limb rather than systemically, acceleration of growth rate during catch-up was only in the limb that received the treatment (14). This supports the hypothesis that the response in catch-up growth is "intrinsic to the growth plate," that it involves interruption of a prepatterned program of growth, that the program can delayed, but that ultimately an intrinsic program determines the final length of a bone, a so-called "sense of size" $(48,49)$. Further support for such an intrinsic program within the growth plate comes from experiments involving transplantation of growth plates. The transplanted growth plate maintains the growth rate of the donor and not of the recipient, suggesting that the transplanted growth plate carries its own program or cadence for continuing growth, despite changes in the systemic hormonal environment (50).

Models involving fasting go beyond the concept of catch-up growth as intrinsic to the growth plate and support the concept that catch-up growth involves the full panoply of variables during the differentiation cascade of the growth plate chondrocyte in the postnatal animal. Some of these are determined at the time of embryonic patterning of the bone and others are regulated by the interplay of systemic and autocrine/paracrine regulators acting throughout the period of growth. This is consistent with considering that basic mechanisms of catch-up growth are regulated through a systemic response under neuroendocrine control, with $\mathrm{GH}$ as a primary target since, in the presence of normal thyroid functioning, GH and IGF-1 constitute the primary hormonal axis of postnatal growth $(51,52)$. The degree of mismatch between the target size and the actual size is compensated for by adjusting growth rate integrated at the level of the nervous system, as originally proposed by Tanner $(32,53)$.

In support of this, studies have found decreases in serum $\mathrm{GH}$ and IGF-1 levels during fasting, and increases during the catch-up period $(5,23,28,54)$. However, a study in 5-wk-old rabbits that were fasted for $2 \mathrm{~d}$ demonstrated that although serum IGF-1 levels fell during the fast, serum GH levels rose. In the growth plate, the IGF-1 system was up-regulated through up-regulation of mRNA for both the GH receptor and IGF-1 (3). A study of young fasted rats indicated that, when giving additional $\mathrm{GH}$ in the postfasting catch-up period, tibial growth in male rats could not be enhanced above the rate involved in "normal" catch-up (26). These authors hypothesized that tibial growth in the catch-up period already was maximal for rats of that age. Although the specifics of the response still needs further investigation, there is a basic consensus that GH-mediated changes in growth rate are involved in the catch-up response to short-term fasting, regulated through a systemic response.

Leptin, the product of the $o b$ gene synthesized in adipose tissue, serves as an afferent signal in a negative feedback loop regulating the size of adipose tissue mass $(7,8,23,55)$. It has been demonstrated that, although leptin given to normal adult rats does not lead to a change in spontaneous GH secretion, 
leptin given to fasted rats causes a reversal of the inhibitory effect fasting causes on GH secretion $(6,56)$. Therefore, leptin can act as a metabolic signal connecting adipose tissue with the GH axis, and may have different roles during normal homeostasis and during fasting. When adult rats or mice were fasted for 1-3 d, there was a sharp decrease in the amount of leptin mRNA in adipose tissue $(57,58)$. Another study demonstrated reduction in the amplitude of leptin pulsatile secretion in rats after a 48-h fast, manifested by decreased plasma levels (59). Therefore, leptin may act as a circulating feedback signal to regulate general energy homeostasis by reflecting adipose tissue stores. Although metabolic changes mediated by leptin are not completely understood, the leptin mediated effects have always been related to weight regulation and a set-point involved with body weight (60). It is interesting to speculate that leptin, acting either directly through its own receptors on growth plate chondrocytes $(61,62)$, or indirectly through either GH or thyroxin (51), may be an important mediator of bone elongation during catch-up growth. This would be a significant area for future investigation.

A continuation of this study will use stereological analyses of chondrocytic performance parameters to analyze chondrocytic responses during the differentiation cascade resulting in increased rate of growth, as has been done in our previous studies using models of several different altered metabolic or biomechanical states $(34,38)$. It will test the hypothesis that the reduced rate of elongation after food deprivation as well as the increased rate after return to normal nutrition are regulated by altering the rate chondrocytes proceed through their differentiation cascade, amplified by volume changes of the hypertrophic chondrocyte. This would be consistent with a response mediated by changes in the GH/IGF-1 axis (39).

Acknowledgments. The authors thank Stephanie Solazzo, Larry Twohig, Michelle Lenox, and Benjamin Benander for technical asistance. We also thank Cherrill Wallen for secretarial assistance.

\section{REFERENCES}

1. Thissen J, Ketelslegers J, Underwood L 1994 Nutritional regulation of the insulin-like growth factors. Endocr Rev 15:80-101

2. Donovan S, Atilano L, Hintz R, Wilson D, Rosenfeld R 1991 Differential regulation of the insulin-like growth factors (IGF-I and -II) and IGF binding proteins during malnutrition in the neonatal rat. Endocrinology 129:149-157

3. Heinrichs C, Colli M, Yanovski JA, Laue L, Gerstl NA, Kramer AD, Uyeda JA, Baron J 1997 Effects of fasting on the growth plate: systemic and local mechanisms. Endocrinology 138:5359-5365

4. Mosier H, Jansons R, Dearden L 1985 Increased secretion of growth hormone in rats undergoing catch-up growth after fasting. Growth 49:346-353

5. Mosier Jr HD, Jansons RA 1976 Growth hormone during catch-up growth and failure of catch-up growth in rats. Endocrinology 98:214-219

6. Carro E, Señaris R, Considine RV, Casanueva FF, Dieguez C 2061997 Regulation of in vivo growth hormone secretion by leptin. Endocrinology 138:2203-2206

7. Ahima RS, Prabakaran D, Mantzoros C, Qu D, Lowell B, Maratos-Flier E, Flier JS 1996 Role of leptin in the neuroendocrine response to fasting. Nature 382:250-252

8. Janeková, R 2001 The role of leptin in human physiology and pathophysiology. Physiol Res 50:443-459

9. Boersma B, Wit JM 1997 Catch-up growth. Endocr Rev 18:646-661

10. Sands J, Dobbing J, Gratrix C 1979 Cell number and cell size: organ growth and development and the control of catch-up growth in rats. Lancet 2:503-505

11. Williams JPG, Tanner JM, Hughes PCR 1974 Catch-up growth in female rats after growth retardation during the suckling period: comparison with males. Pediatr Res $8: 157-162$

12. Williams J, Hughes P 1975 Catch-up growth in rats undernourished for different periods during the suckling period. Growth 39:179-193
13. Prader A, Tanner JM, von Harnack GA 1963 Catch-up growth following illness or starvation. J Pediatr 62:646-659

14. Gafni RI, Weise M, Robrecht DT, Meyers JL, Barnes KM, De-Levi S, Baron J 2001 Catch-up growth is associated with delayed senescence of the growth plate in rabbits. Pediatr Res 50:618-623

15. Dearden LC, Mosier Jr HD 1974 Growth retardation and subsequent recovery of the rat tibia, a histochemical, light, and electron microscopic study. I. After propylthiouracil treatment. Growth 38:253-275

16. Mosier Jr HD 1969 Allometry of body weight and tail length in studies of catch-up growth. Growth 33:319-330

17. Mosier Jr HD 1971 Failure of compensatory (catch-up) growth in the rat. Pediatr Res 5:59-63

18. Sinha YN, Wilkins JN, Selby F, VanderLaan WP 1973 Pituitary and serum growth hormone during undernutrition and catch-up growth in young rats. Endocrinology 92:1768-1771

19. Robson H 1999 Bone growth mechanisms and the effects of cytotoxic drugs. Arch Dis Child 81:360-364

20. Stephan JK, Chow B, Frohman LA, Chow BF 1971 Relationship of growth hormone to the growth retardation associated with maternal dietary restriction. J Nutr 101:1453-1458

21. Woodall SM, Breier BH, Johnston BM, Gluckman PD 1996 A model of intrauterine growth retardation caused by chronic maternal undernutrition in the rat: effects on the somatotrophic axis and postnatal growth. J Endocrinol 150:231-242

22. Houdijk ECAM, Engelbregt MJT, Popp-Snijders C, Delemarre-vd Waal HA 2000 Endocrine regulation and extended follow up of longitudinal growth in intrauterine growth-retarded rats. J Endocrinol 166:599-608

23. Engelbregt MJT, vanWeissenbruch MM, Popp-Snijders C, Lips P, Delemarre-van de Waal HA 2001 Body mass index, body composition, and leptin at onset of puberty in male and female rats after intrauterine growth retardation and after early postnatal food restriction Pediatr Res 50/4:474-478

24. Faust IM, Johnson PR, Hirsch J 1980 Long-term effects of early nutritional experience on the development of obesity in the rat. J Nutr 110:2027-2034

25. Dearden LC, Jr Mosier HD 1974 Growth retardation and subsequent recovery of the rat tibia, a histochemical, light, and electron microscopic study. II. After fasting. Growth 38:277-294

26. Rol de Lama MA, Pérez-Romero A, Tresguerres JAF, Hermanussen M, Ariznavarreta C 2000 Recombinant human growth hormone enhances tibial growth in peripubertal female rats but not in males. Eur J Endocrinol 142:517-523

27. Widdowson EM, McCance RA 1963 The effect of finite periods of undernutrition at different ages on the composition and subsequent development of the rat. Proc R Soc London B Biol Sci 158:329-342

28. Hermanussen M, Rol de Lama MA, Romero AP, Ruiz CA, Burmeister J, Tresguerres JAF 1996 Differential catch-up in body weight and bone growth after short-term starvation in rats. Growth Regul 6:230-237

29. Bruno JF, Olchovsky D, White JD, Leidy JW, Song J, Berelowitz M 1990 Influence of food deprivation in the rat on hypothalamic expression of growth hormonereleasing factor and somatostatin. Endocrinology 127:2111-2116

30. Hughes PLR, Turner JM 1970 The assessment of skeletal maturity in the growing rat. J Anat 106:371-402

31. Jaffe CA, Ocampo-Lim B, Guo W, Krueger K, Sugahara I, DeMott-Friberg R, Bermann M, Barkan AL 1998 Regulatory mechanisms of growth hormone secretion are sexually dimorphic. J Clin Invest 102:153-164

32. Tanner JM 1963 Regulation of growth in size from mammals. Nature 199:845-850

33. Treharne RW, Brighton CT 1979 The use and possible misuse of tetracycline as a vital stain. Clin Orthop 140:240-246

34. Wilsman NJ, Farnum CE, Leiferman EM, Fry M, Barreto C 1996 Differential growth by growth plates as a function of multiple parameters of chondrocytic kinetics. J Orthop Res 14:927-936

35. Hunziker EB, Schenk R 1989 Physiological mechanisms adopted by chondrocytes in regulating longitudinal bone growth in rats. J Physiol 414:55-71

36. Hunziker EB, Herrmann W, Schenk R 1983 Ruthenium hexamine trichloride (RHT)mediated interactions between the plasmalemmal components and pericellular matrix proteoglycans is responsible for the preservation of chondrocytic plasma membrane in situ during cartilage fixation. J Histochem Cytochem 31:717-727

37. Hunziker EB, Schenk RK, Cruz-Orive L-M 1987 Quantitation of chondrocyte performance in growth-plate cartilage during longitudinal growth. J Bone Joint Surg Am 69:162-173

38. Farnum CE, Nixon A, Lee AO, Kwan DT, Belanger L, Wilsman NJ 2000 Quantitative three-dimensional analysis of chondrocytic kinetic responses to short-term stapling of the rat proximal tibial growth plate. Cells Tissues Organs 167:247-258

39. Farnum CE, MacLeod M 1997 Growth hormone effects on chondrocytic activity in relationship to postnatal longitudinal growth of the proximal tibia. Proceedings of the Orthopedic Research Society 43:132

40. Humphrey CD, Pittman FE 1974 A simple methylene blue-Azure II-basic fuchsin stain for epoxy tissue sections. Stain Technol 419:9-10

41. Hunziker EB 1994 Mechanisms of longitudinal growth and its regulation by growth plate chondrocytes. Microsc Res Tech 28:505-519

42. Baron J, Klein KO, Colli MJ, Yanovski JA, Novosad JA, Bacher JD, Cutler Jr GB 1994 Catch-up growth after glucocorticoid excess: a mechanism intrinsic to the growth plate. Endocrinology 135:1367-1371

43. Farnum CE 1994 Differential rates of growth of long bones. In: Hall BK (ed) Bone, Vol 8, Mechanisms of Bone Development and Growth. CRC Press, Boca Raton, FL, pp 193-222

44. Farnum CE, Wilsman NJ 2001 Converting a differentiation cascade into longitudinal growth: stereology and analysis of transgenic animals as tools for understanding growth plate function. Curr Opin Orthop 12:428-433 
45. Farnum CE, Wilsman NJ 1998 Growth plate cellular function. In: Buckwalter JA Ehrlich MG, Sandell LJ, Trippel SB (eds) Skeletal Growth and Development. American Academy of Orthopaedic Surgeons, Rosemont, IL, pp 203-223

46. Tyrcha J 2001 Age-dependent cell cycle models. J Theor Biol 213:89-101

47. Campisi J 1996 Replicative senescence: an old lives' tale? Cell 84:497-500

48. Albanese A, Kewley GD, Long A, Pearl KN, Robins DG, Stanhope R 1994 Oral treatment for constitutional delay of growth and puberty in boys: a randomised trial of an anabolic steroid or testosterone undecanoate. Arch Dis Child 71:315-317

49. Joss EE, Schmidt HA, Zuppinger KA 1989 Oxandrolone in constitutionally delayed growth, a longitudinal study up to final height. J Clin Endocrinol Metab 69:11091115

50. Stevens DG, Boyer MI, Bowen CVA 1999 Transplantation of epiphyseal plate allografts between animals of different ages. J Pediatr Orthop 19:398-403

51. Siebler T, Robson H, Shalet SM, Williams, GR 2001 Glucocorticoids, thyroid hormone and growth hormone interactions; implications for the growth plate. Horm Res 56(suppl 1):7-12

52. Tsatsoulis A, Siamopoulou A, Petsoukis C, Challa A, Bairaktari E, Seferiadis K 1999 Study of growth hormone secretion and action in growth-retarded children with juvenile chronic arthritis (JCA). Growth Horm IGF Res 9:143-149

53. Tanner JM 1981 Catch-up growth in man. Br Med Bull 37:233-238

54. Mosier HD, Catalano RA, Serxner S, Mosier C, Jansons RA 1987 Frequency of growth hormone secretion associated with catch-up growth in the rat detected by time domain (box-jenkins) analysis. Growth 51:249-260
55. Cusin I, Sainsbury A, Doyle P, Rohner-Jeanrenaud F, Jeanrenaud P 1995 The $o b$ gene and insulin: A relationship leading to clues to the understanding of obesity. Diabetes 44:1467-1470

56. Flier JS, Harris M, Hollenberg AN 2000 Leptin, nutrition, and the thyroid: the why, the wherefore, and the wiring. J Clin Invest 105:859-861

57. Mizuno TM, Bergen H, Funabashi T, Kleopoulos SP, Zhong Y-G, Bauman WA, Mobbs CV 1996 Obese gene expression: reduction by fasting and stimulation by insulin and glucose in lean mice, and persistent elevation in acquired (diet-induced) and genetic (yellow agouti) obesity. Proc Natl Acad Sci USA 93:3434-3438

58. Frederich RC, Löllmann B, Hamann A, Napolitano-Rosen A, Kahn BB, Lowell BB, Flier JS 1995 Expression of ob mRNA and its encoded protein in rodents. Impact of nutrition and obesity. J Clin Invest 96:1658-1663

59. Bagnasco M, Kalra PS, Kalra, SP 2002 Ghrelin and leptin discharge in fed and fasted rats. Endocrinology 143:726-729

60. Friedman JM 1998 Leptin, leptin receptors, and the control of body weight. Nutr Rev 56:S38-S46

61. Hoggard N, Hunter L, Duncan JS, Williams LM, Trayhurn P, Mercer JG 1997 Leptin and leptin receptor mRNA and protein expression in the murine fetus and placenta. Proc Natl Acad Sci U S A 94:11073-11078

62. Kume K, Satomura K, Nishisho S, Kitaoka E, Yamanouchi K, Tobiume S, Nagayama, M 2002 Potential role of leptin in endochondral ossification. J Histochem Cytochem 50:159-169 